Research Paper

\title{
Scaling Laws of Rough Turbulent Flows from Turbulence Phenomenology: An Overview and a New Application
}

\author{
SK ZEESHAN ALI and SUBHASISH DEY* \\ Department of Civil Engineering, Indian Institute of Technology Kharagpur, West Bengal 721 302, India
}

(Received on 05 April 2016; Accepted on 15 April 2016)

\begin{abstract}
In this paper, the scaling laws of rough turbulent flows stemming from the phenomenological theory of turbulence are reviewed. It is revealed that the scaling of the well-known empirical formulae can also be obtained by means of the phenomenological theory. The phenomenological theory thus enables to provide an insight into the physics of the flows of the problems arising from turbulence. As a new thought, the famous scaling laws of the velocity distributions, the logarithmic law and the power law of the wall, are also sought applying the same theory.
\end{abstract}

Keywords: Fluid Mechanics; Hydrodynamics; Turbulence; Turbulent Flow

\section{Introduction}

Turbulence, the unrest phenomenon of fluid in nature, is believed to be one of the most challenging and intricate aspects of fluid mechanics and applied hydrodynamics. Although considerable endeavors were made to study the behavioural phenomena of turbulent flows over rough boundaries (Raupach et al., 1991; Jiménez, 2004), the turbulence phenomena remain the least understood aspects in fluid mechanics. In many physical problems concerning the rough turbulent flows, researchers are interested to seek different scaling laws. In doing so, several empirical and semi-empirical laws based on the experimental data were proposed. However, there remains an important question that to what extent these empirical or semi-empirical laws are legitimate from the perspective of turbulence. This question can now be answered by applying the phenomenological theory of turbulence.

The phenomenological theory of turbulence originated from Kolmogorov's (1941) philosophical thoughts on the scaling laws of fully developed steady state turbulence. Although Kolmogorov's original ideas were based on unconfirmed, but plausible doctrines of the homogeneous and isotropic turbulence, several studies after Kolmogorov's (1941) theory revealed that the Kolmogorov's scaling laws are still valid for the non-homogeneous and anisotropic turbulent flows (Knight and Sirovich, 1990; Moser, 1993; Saddoughi and Veeravalli, 1994; Gioia and Chakraborty, 2006). Frisch (1995) defined the phenomenology of turbulence as a shorthand system that allows to obtain the scaling laws of fully developed steady state turbulence in a much simpler way at the price of less heuristic arguments. Another advantage of the phenomenological theory is that it associates the dependent and the independent variables by means of turbulence scaling laws, in which each of the independent variables possesses a single unique free exponent. In other words, the phenomenological theory controls the degrees of freedom of the free exponents. However, in case of empirical or semi-empirical laws describing the turbulent flow phenomena, the free exponents of the independent variables are determined from the regression analyses using the experimental data. As a consequence, when the empirical or semiempirical laws proposed by different researchers are applied to analyze the same problem, discrepancies in results among the different formulae are often found. Although the phenomenological theory was primarily used to model the turbulent flows (Frisch, 
1995; Sreenivasan and Antonia, 1997), in this century, it is however established that the applications of the phenomenological theory provide a simple, but scientific, framework to seek the scaling laws of rough turbulent flows. Therefore, due to its immense importance in finding the scaling laws, several researchers applied the phenomenological theory to seek the scaling laws of rough turbulent flows. In this paper, a review of the important studies on the phenomenological theory to seek the scaling laws of rough turbulent flows is presented. Then, the new scaling laws of the velocity distributions, the logarithmic law and the power law of the wall, over solid boundaries are proposed from the perspective of the phenomenological theory.

\section{Applications of Turbulence Phenomenology}

\section{The Scaling Law of Mean Velocity}

Manning's empirical equation is widely used to obtain the mean velocity $U_{m}$ of a zero-pressure gradient rough turbulent flow in open channels. It is expressed as

$$
U_{m}=\frac{1}{n} R_{b}^{2 / 3} S_{0}^{1 / 2}
$$

where $n$ is the Manning roughness coefficient, $R_{b}$ is the hydraulic radius and $S_{0}$ is the bottom slope of the channel. The hydraulic radius $R_{b}$ is defined as the ratio of channel cross-sectional area to its wetted perimeter. For instance, for a rectangular channel of width $b$ and flow depth $h$, hydraulic radius is $R_{b}=b h /$ $(b+2 h)$. Thus, for a wide rectangular channel $\left(b \gg h\right.$ ), one can approximately consider $R_{b} \approx h$.

Gioia and Bombardelli (2002) were the first to derive the Manning equation from the perspective of the phenomenology of turbulence. Before coming to the derivation of Eq. (1), it is intended to understand as to what extent Eq. (1) can be obtained from the dimensional analysis and similarity methods. To start with, four plausible variables, $U_{m}, R_{b}, k_{s}$ and $g S_{0}$, are considered. Here, $k_{s}$ is the boundary roughness height and $g$ is the acceleration due to gravity. Thus, the product $g S_{0}$ defines the dynamic component of the gravitational acceleration. In functional form, the equation $f\left(U_{m}, R_{b}, k_{s}, g S_{0}\right)=0$ therefore exists. Applying the Buckingham $\Pi$ theorem (Dey, 2014), one obtains two $\Pi$ parameters: $\Pi_{1}=U_{m} /\left(R_{b} g S_{0}\right)$ and $\Pi_{2}=k_{s} / R_{b}$. Hence, in functional form, one can write

$$
U_{m}=\sqrt{R_{b} g S_{0}} f_{1}\left(\frac{k_{s}}{R_{b}}\right)
$$

In a natural channel or a river, the $k_{s} / R_{b}$-ratio is usually $k_{s} / R_{b} \ll 1$. In fact, there exist two plausible similarities: Complete and incomplete. In case of a complete similarity, $f_{1}\left(k_{s} / R_{b}\right)$ asymptotically reaches a constant value as the value of $k_{s} / R_{b}$-ratio tends to zero. However, this observation is contrary to the measurements, since the mean velocity is dependent on the boundary roughness. On the other hand, in case of an incomplete similarity, Eq. (2) can be written as

$$
U_{m}=C \sqrt{R_{b} g S_{0}}\left(\frac{k_{s}}{R_{b}}\right)^{\beta}+O\left(\frac{k_{s}}{R_{b}}\right)^{\beta}
$$

where $C$ is a coefficient and $\beta$ is a similarity exponent, which cannot be obtained from the dimensional analysis. Comparing Eqs. (1) and (3), one finds that the foremost term of Eq. (3) is consistent with the Manning equation, Eq. (1), if $\beta=1 / 6$. Thus, it results $n=C^{-1} k_{s}^{1 / 6} g^{-1 / 2}$. The " $1 / 6$ " scaling law with $k_{s}$ is in conformity with the measurements by Strickler (Dey, 2014), while the "-1/2" scaling law with $g$ corroborates with the observations of Carr (1979) and Yen (1992). Therefore, to derive Eq. (1), one expects to verify that $\beta=-1 / 6$.

Consider a fully developed rough-turbulent stream (hydraulically rough flow regime) flowing through a rectangular channel as sketched in Fig. 1. The mean flow depth is $h$. The dynamic component of the gravitational force per unit length of the channel is expressed as $F_{g}=\rho_{f} g S_{0} b h$, where $\rho_{f}$ is the mass density of fluid.

Now, a length scale $l$ is considered lying between the integral length scale $L$ and the Kolmogorov dissipation length scale $\eta\left[=\left(v^{3} / \varepsilon\right)^{1 / 4}\right.$, where $v$ is the coefficient of kinematic viscosity and $\varepsilon$ is the turbulent kinetic energy (TKE) dissipation rate]. The integral length scale $L$ can be scaled with the external length scale, for example, the flow depth $h$ or more 


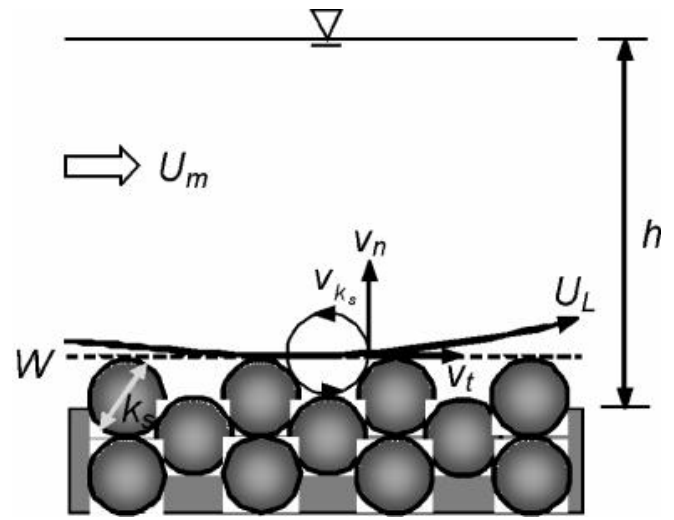

Fig. 1: Schematic of rough turbulent flow in an open channel

specifically the hydraulic radius $R_{b}$. If $v_{l}$ is the characteristic velocity scale that corresponds to $l$, the circulation time $t_{l}$ is $t_{l} \sim l / v_{l}$, where the symbol " $\sim$ denotes the "scales with". Subsequently, the energy flux $E_{l}$ transferred from the scales $l$ to the smaller ones is given by $E_{l} \sim v_{l}^{2} / t_{l} \sim v_{l}^{3} / l$. Since in the inertial subrange, the TKE production rate and the TKE dissipation rate $\varepsilon$ balance each other, one can obtain $E_{l}=\varepsilon \sim v_{l}^{3} / l$. However, for $l \sim L$, it yields $\varepsilon \sim U_{L}^{3} / L$, where $U_{L}$ is the velocity scale corresponding to $L$. It may be noted that the $U_{L}$ can be scaled with the external velocity scale, for example, the mean flow velocity $U_{m}$. Thus, the above phenomenological consideration yields $v_{l} \sim U_{L}(l / L)^{1 / 3} \sim U_{m}\left(l / R_{b}\right)^{1 / 3}$.

The Reynolds shear stress $\tau_{t}$ developed due to the localized eddies in the immediate vicinity of the boundary at an imaginary wetted surface $W$ tangential to the summit of the boundary roughness elements is $\tau_{t}=\rho_{f} \overline{v_{t} v_{n}}$ (Pope, 2000), where $v_{t}$ and $v_{n}$ are the velocity fluctuations tangential and normal to $W$, respectively and the overbar denotes the timeaveraged value. Using the scaling law $v_{l} \sim U_{m}(l /$ $\left.R_{b}\right)^{1 / 3}$, the velocity scale of the localized eddies $(l \sim$ $k_{s}$ ) follows:

$$
v_{l} \sim v_{k_{s}} \sim U_{m}\left(\frac{k_{s}}{R_{b}}\right)^{1 / 3}
$$

To determine $v_{t}$, Gioia and Bombardelli (2002) considered $v_{t} \sim U_{L} \sim U_{m}$, since the eddies of all sizes can induce shear tangential to $W$. However, $v_{n} \sim v_{k_{s}}$ because the smaller eddies $\left(l \sim k_{s}\right)$ provide substantial contribution to the momentum flux normal to $W$, while the larger eddies $\left(l \gg k_{s}\right)$ contribute feebly. Thus,

$$
\tau_{t} \sim \rho_{f} U_{m} v_{k_{s}} \sim \rho_{f} U_{m}^{2}\left(\frac{k_{s}}{R_{b}}\right)^{1 / 3}
$$

The streamwise component of force per unit length acting on the imaginary wetted surface is $F_{t}=$ $\tau_{t}(b+2 h)$. Applying the momentum balance, one obtains $F_{t}=F_{g}$. Therefore,

$$
\begin{aligned}
& \rho_{f} U_{m}^{2}\left(\frac{k_{s}}{R_{b}}\right)^{1 / 3}(b+2 h) \sim \rho_{f} g S_{0} b h \Rightarrow \\
& U_{m} \sim \sqrt{R_{b} g S_{0}}\left(\frac{k_{s}}{R_{b}}\right)^{-1 / 6}
\end{aligned}
$$

Thus, Eq. (6) resembles to the foremost term of Eq. (3) with $\beta=-1 / 6$. This completes the derivation.

In this context, it is pertinent to mention that if the boundary roughness height $k_{s}$ reduces, the mean velocity $U_{m}$ increases due to the reduction in the momentum transfer. Subsequently, when the boundary roughness height $k_{s}$ approaches the Kolmogorov dissipation length scale $\eta\left(k_{s} \rightarrow \eta\right)$, using $\varepsilon \sim U_{m}^{3} / R_{b}$, one obtains

$$
\frac{\eta}{R_{b}} \sim \operatorname{Re}^{-3 / 4}
$$

where $\operatorname{Re}\left(=U_{m} R_{b} / v\right)$ is the Reynolds number. Therefore, Eq. (7) reduces to

$$
U_{m} \sim \sqrt{R_{b} g S_{0}} \operatorname{Re}^{1 / 8}
$$

The presence of Re in Eq. (8) reveals that the momentum transfer is viscous in the limit $k_{s} \rightarrow \eta$. Introducing the resistance coefficient $f=R_{b} g S_{0} / U_{m}^{2}$, one can rewrite Eq. (8) as

$$
f \sim \operatorname{Re}^{-1 / 4}
$$

which is the well-known empirical scaling of Blasius for hydraulically smooth channels (Dey, 2014). 
The Scaling Law of Scour Depth Caused by Localized Turbulent Flows

When a turbulent jet impinges into a pool having a uniform flow depth, a turbulent cauldron is formed under the action of the jet. Due to the energetics of the jet, the turbulent cauldron starts to scour the bed to form a pothole as depicted in Fig. 2. The depth of the pothole increases until a dynamic equilibrium is established between the sediment bed and the turbulent cauldron. For such a problem, researchers are interested to find the equilibrium depth of the pothole for a given power of the jet. An extensive survey of empirical equations for the equilibrium depth of the pothole was done by Dey (2014). These empirical equations can be written in a generalized form as

$$
D=C q^{\alpha_{q}} h_{0}^{\alpha_{h_{0}}} g^{\alpha_{g}} d^{\alpha_{d}}\left(\frac{\rho_{f}}{\rho_{s}-\rho_{f}}\right)^{\alpha_{\rho}}
$$

where $D$ is the sum of the depth of the pothole $d_{s}$ and the depth of the pool $h$, that is $D=d_{s}+h, q$ is the discharge of the jet per unit thickness, $h_{0}$ is the head of the jet, $d$ is the characteristic size of the sediment particles, $\rho_{s}$ is the mass density of sediment particles and $\alpha_{q}, \alpha_{h_{0}}, \alpha_{g}, \alpha_{d}$ and $\alpha_{\rho}$ are the empirical exponents.

Gioia and Bombardelli (2005) and Bombardelli and Gioia (2006) studied the local scour due to turbulent jet applying the phenomenology of turbulence. Before predicting the equilibrium scour

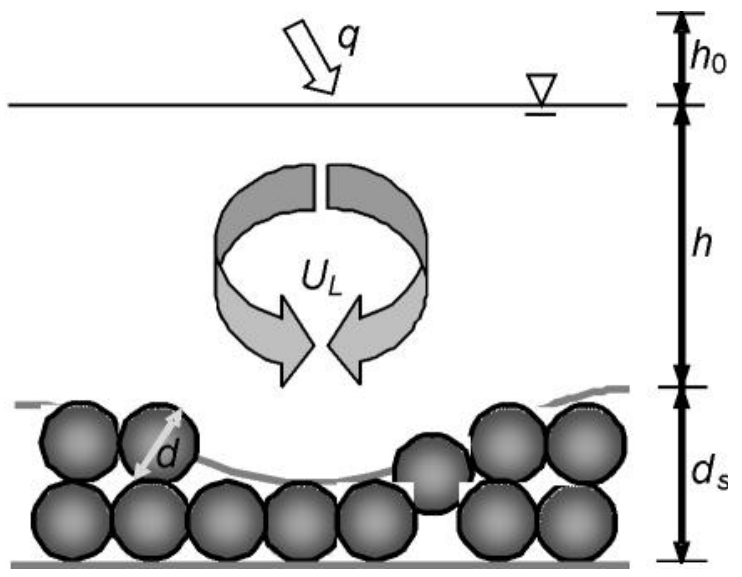

Fig. 2: Schematic of local scour due to turbulent jet depth, it is appropriate to examine to what extent Eq. (10) can be obtained from the dimensional analysis and similarity methods. To start, six plausible variables $D, \rho_{f}, g, \rho_{s}, d$ and $P$ are considered, where $P$ is the power of the jet per unit thickness. Thus, in functional form, the equation $f\left(D, \rho_{f}, g, \rho_{s}, d, P\right)=0$ exists. Applying the Buckingham $\Pi$ theorem, one obtains three $\Pi$ parameters: $\Pi_{1}=P /\left(\rho_{f} g^{3 / 2} D^{5 / 2}\right), \Pi_{2}=\rho_{s} / \rho_{f}$ and $\Pi_{3}=d / D$. Hence, one can write

$$
P=\rho_{f} g^{3 / 2} D^{5 / 2} f_{2}\left(\frac{d}{D}, \frac{\rho_{s}}{\rho_{f}}\right)
$$

In case of a complete similarity, $f(d / D)$ asymptotically reaches a constant value as the value of $d / D$ tends to zero. For such a scenario, $D$ is independent of $d$ for $d / D \ll 1$. However, this observation is contrary to the measurements since the values of the empirical exponent $\alpha_{d}$ proposed so far are different from zero. On the other hand, in case of an incomplete similarity, Eq. (11) can be written as

$$
P=\rho_{f} g^{3 / 2} D^{5 / 2}\left(\frac{d}{D}\right)^{\beta} f_{3}\left[\frac{\rho_{s}}{\rho_{f}}+O\left(\frac{d}{D}\right)^{\beta}\right]
$$

Substituting the foremost term of Eq. (12) in Eq. (11) and rearranging yield

$$
D=C q^{\alpha_{q}} h_{0}^{\alpha_{h_{0}}} g^{\alpha_{g}} d^{\alpha_{d}} f_{4}\left(\frac{\rho_{s}}{\rho_{f}}\right)
$$

where $\alpha_{q}=\alpha_{h_{0}}=2 /(5-2 \beta), \alpha_{q}=-1 /(5-2 \beta), \alpha_{d}=$ $-2 \alpha /(5-2 \beta)$ and $f_{4}=1 /\left[C f_{3}^{2 /(5-2 \beta)}\right]$. It may be noted that Eq. (13) has the similar form as Eq. (10). Moreover, the empirical exponents are related to each other via a single free parameter $\alpha$, which can be determined from the phenomenology of turbulence.

From the phenomenological perspective as already derived before, one obtains $v_{l} \sim U_{L}(l / L)^{1 / 3}$. Considering $L \sim D$, yields $\varepsilon \sim U_{L}^{3} / D$. The power of the jet per unit thickness is $P=q \rho_{f} g h_{0}$. Further, $P$ 
can be expressed as $P=\varepsilon M$, where $M$ is the mass per unit thickness of the turbulent cauldron. The $M$ can be scaled as $M \sim \rho_{f} D^{2}$. Thus, one obtains

$$
U_{L} \sim\left(q g \frac{h_{0}}{D}\right)^{1 / 3}
$$

The Reynolds shear stress $\tau_{t}$ developed due to the localized eddies at an imaginary wetted surface $W$ tangential to the summit of the sediment particles at the surface of the pothole is $\tau_{t}=\rho_{f} \overline{v_{t} v_{n}} \sim \rho_{f} U_{L} v_{l}$. The velocity scale of the localized eddies $(l \sim d)$ follows: $v_{l} \sim v_{d} \sim U_{L}(d / D)^{1 / 3}$. Thus, using Eq. (14) yields

$$
\tau_{t} \sim \rho_{f} \frac{\left(q g h_{0}\right)^{2 / 3} d^{1 / 3}}{D}
$$

When the depth $d_{s}$ of the pothole increases, the size $D$ of the turbulent cauldron increases at the same time. As a consequence, the boundary shear stress exerted on the pothole surface reduces due to the reduced velocity resulting from the increased crosssectional area of flow. Eventually, the scour ceases when the boundary shear stress becomes equal to the threshold boundary shear stress for the incipient motion of sediment particles. The threshold boundary shear stress $\tau_{c}$ for the hydraulically rough flow regime follows: $\tau_{c} \sim\left(\rho_{s}-\rho_{f}\right) g d$. Thus, substituting Eq. (15) and $\tau_{c} \sim\left(\rho_{s}-\rho_{f}\right) g d$ into $\tau_{t} \sim \tau_{c}$ yields

$$
D \sim q^{2 / 3} h_{0}^{2 / 3} g^{-1 / 3} d^{-2 / 3}\left(\frac{\rho_{f}}{\rho_{s}-\rho_{f}}\right)
$$

Equation (16) represents the complete theoretical expression for the depth of the pothole as $d_{s}=D-h$. Comparing Eqs. (16) and (13) reveals $\alpha_{q}=\alpha_{h_{0}}=2 / 3$ $\alpha_{g}=-1 / 3, \alpha_{d}=-2 / 3$ with the similarity exponent $\beta=$ 1 .

Manes and Brocchini (2015) also applied the phenomenology of turbulence to study the local scour around a solid cylinder embedded in a sediment bed. Applying the same concept as discussed, they determined the scaling law of the equilibrium scour depth as a function of flow properties, sediment size and cylinder diameter. From dimensional analysis, the mass of the largest eddy is expressed as $M \sim \rho_{f} L^{3}$. The TKE dissipation rate $\varepsilon$ is obtained as the power $P$ associated with the largest eddy divided by the fluid mass $M$ contained within the characteristic volume. The power $P$ is obtained as the work done by a drag force $F_{D}$ on the cylinder against the mean flow. Hence, the power $P=F_{D} U_{m}$. The drag force $F_{D}$ is computed as $F_{D}=0.5 \rho_{f} C_{D} a L U_{m}^{2}$, where $C_{D}$ is the drag coefficient, $a$ is the diameter of cylinder and the product $a L$ represents the frontal area of the cylinder exposed to scouring. Thus

$\varepsilon=\frac{P}{M} \sim \frac{C_{D} a U_{m}^{3}}{L^{2}} \sim \frac{U_{L}^{3}}{L} \Rightarrow U_{L} \sim U_{m}\left(\frac{C_{D} a}{L}\right)^{1 / 3}$

Manes and Brocchini (2015) considered that the length scale $L$ of the largest eddy can be scaled with the equilibrium scour depth $d_{s}\left(L \sim d_{s}\right)$. Therefore, the Reynolds shear stress $\tau_{t}$ is obtained as

$$
\tau_{t} \sim \rho_{f} U_{L}^{2}\left(\frac{d}{L}\right)^{1 / 3} \sim U_{m}^{2}\left(\frac{C_{D} a}{d_{s}}\right)^{2 / 3} d_{s}^{1 / 3}
$$

At an equilibrium condition, the Reynolds shear stress $\tau_{t}$ reaches the threshold boundary shear stress $\tau_{c}\left[\sim\left(\rho_{s}-\rho_{f}\right) g d\right]$. Thus, substituting Eq. (18) and $\tau_{c} \sim$ $\left(\rho_{s}-\rho_{f}\right) g d$ into $\tau_{t} \sim \tau_{c}$ yields

$$
\frac{d_{s} g}{U_{m}^{2}} \sim\left(\frac{\rho_{f}}{\rho_{s}-\rho_{f}}\right) C_{D}^{2 / 3}\left(\frac{a}{d}\right)^{2 / 3}
$$

\section{New Application of Turbulence Phenomenology}

\section{The Logarithmic Law of the Wall}

The logarithmic law of the wall, commonly called the log-law, is widely used to describe the velocity distribution for the turbulent wall bounded flows and believed to be one of the marvelous contributions toward modern fluid dynamics (Bradshaw and Huang, 1995). The genesis of log-law was due to von Kármán (Schlichting, 1979), who invented this law from the idea of constant Reynolds shear stress in the turbulent wall shear layer. The log-law states that the local timeaveraged flow velocity $\bar{u}$ (scaled by the friction 
velocity $u_{*}$ ) at a specific point is proportional to the logarithm of the vertical distance $z$ (scaled by the zero velocity level $z_{0}$ ) of that point from the wall. In mathematical form, it is expressed as $u^{+} \sim \ln z^{+}$, where $u^{+}$is $\bar{u} / u_{*}$ and $z^{+}$is $z / z_{0}$. The above phenomenological constant is denoted by $\kappa^{-1}$, where $\kappa$ is the von Kármán constant, which is believed to be a universal constant in flow over a solid wall (Dey, 2014). However, the non-universal behaviour of log-law due to the deviation of $\kappa$ from its universal value was also reported in literature (Bradshaw and Huang, 1995; Panton, 2005; George, 2007; Dey, 2014). Here, we show that the existence of log-law in the turbulent wall shear layer from the phenomenology of near-wall turbulence.

The characteristics of the localized eddy in close proximity of the wall are governed by two key parameters: The local stress $s_{l}(\sim d \bar{u} / d z)$ and its gradient $\dot{s}_{l}$, where dot denotes the derivative with respect to $z$. We also consider that the tangential and normal velocity fluctuations are of the same order $\left(v_{t} \sim v_{n}\right)$ within the turbulent wall shear layer (Panton, 2005). Thus, in functional forms, one can write $l$ $\sim F\left(s_{l}, \dot{s}_{l}\right)$ and $v_{t}\left(\sim v_{n}\right) \sim G\left(s_{l}, \dot{s}_{l}\right)$. Simple dimensional analysis shows that $l \sim s_{l} \dot{s}_{l}^{-1}$ and $u_{t}(\sim$ $\left.u_{n}\right) \sim s_{l}^{2} \dot{s}_{l}^{-1}$. Therefore, the Reynolds shear stress $\tau_{t}$ developed within the turbulent wall shear layer is $\tau_{t}=\rho_{f} \overline{v_{t} v_{n}} \sim \rho_{f} s_{l}^{4} \dot{s}_{l}^{-2}$. The TKE production rate $t_{p}$ is expressed as $t_{p}=\left(\tau / \rho_{f}\right) \times s_{l} \sim s_{l}^{5} \dot{s}_{l}^{-2}$, while the TKE dissipation rate $\varepsilon$ in the turbulent wall shear layer is $\varepsilon$ $\sim u_{*}^{3} / l \sim u_{*}^{3} s_{l}^{-1} \dot{s}_{l}$. In the turbulent wall shear layer, the TKE production rate approximately equals the TKE dissipation rate $\left(t_{p} \approx \varepsilon\right)$, yielding $s_{l}^{5} \dot{s}_{l}^{-2} \sim u_{*}^{3} s_{l}^{-1} \dot{s}_{l}$. Therefore, one obtains $s_{l}^{2} \sim u_{*} \dot{s}_{l}$, and the integration of which results in $s_{l} \sim u_{*} / z$ (neglecting the sign of $s_{l}$ ). Integrating again, one gets $u^{+} \sim \ln z^{+}$, which is the form of the log-law of the wall.

\section{The Power Law of the Wall}

The power law of the wall, commonly called the power-law, is also widely used to describe the velocity distribution for the turbulent wall bounded flows and is regarded as a good approximation of the log-law over the entire boundary layer thickness (Schlichting, 1979). The power-law of velocity distribution states that the local time-averaged flow velocity $\bar{u}$ (scaled by the maximum flow velocity $U_{\max }$ or flow velocity at the edge of the boundary layer) is proportional to the $1 / m$-th power of the vertical distance $z$ (scaled by the boundary layer thickness $\delta$ ). In mathematical form, it is expressed as $\hat{u}=\hat{z}^{1 / m}$, where $\hat{u}$ is $\bar{u} / U_{\max }, \hat{z}$ is $z / \delta$ and $m$ is an exponent of the power-law. To seek the origin of the power-law, a turbulent wall bounded flow having a mean flow velocity $U_{m}$ is considered. The length scale $L$ and velocity scale $U_{L}$ of the largest eddy can be scaled with $\delta$ and $U_{m}$, respectively. At the extremity of the inertial subrange $(l \sim L)$, one gets $\varepsilon \sim U_{m}^{3} / \delta$ and $v_{l} \sim U_{m}(l / \delta)^{1 / 3}$. To determine $v_{t}$ at any vertical distance $z$, it can be assumed that $v_{t} \sim U_{L} \sim$ $U_{m}$ since the larger eddies have a substantial contribution to the momentum flux along the flow direction. However, conceptually $v_{n} \sim v_{l}$ since the smaller eddies have a considerable contribution to the momentum flux normal to the flow direction, while larger eddies have a weak contribution. Thus, at any $z$, one obtains $\tau_{t} \sim U_{m} v_{l} \sim U_{m}^{2}(l / \delta)^{1 / 3}$. Furthermore, the mean flow velocity $U_{m}$ can be scaled with the maximum flow velocity $U_{\max }\left(U_{m} \sim U_{\max }\right)$. Thus, one can write $\tau_{t} \sim U_{\max }^{2}(l / \delta)^{1 / 3} \sim s_{l}^{4} \dot{s}_{l}^{-2}$ (as already obtained from the preceding section). Using $l \sim s_{l} \dot{s}_{l}^{-1}$ the above phenomenological relationship yields $\dot{s}_{l} \sim s_{l}^{11 / 5} \delta^{1 / 5} U_{\max }^{-6 / 5}$, and integration of which produces $s_{l} \sim U_{\max } \delta^{-1 / 6} z^{-5 / 6}$ (neglecting the sign of $s_{l}$ ). Integrating again, one obtains $\hat{u} \sim \hat{z}^{1 / 6}$. This shows the origin of the power law of velocity distribution with $m=6$. Measurements showed that the value of $m$ typically varies from 6 to 10 depending on the flow Reynolds numbers (Schlichting, 1979). The present phenomenological study predicts $m=6$, which is the lower limit of the predicted values.

\section{Conclusions}

The scaling laws of rough turbulent flows obtained from the phenomenological theory of turbulence are 
reviewed. A close agreement between these scaling laws and the empirical laws reveals that the phenomenological theory provides an easy but scientific means to find the scaling laws of rough turbulent flows. The theory is capable to capture the essential features of the physics of the flows in the context of the momentum transfer. In addition, the new scaling laws of the velocity distributions (the logarithmic law and the power law of the wall) are explored by applying the same theory.

\section{Notation}

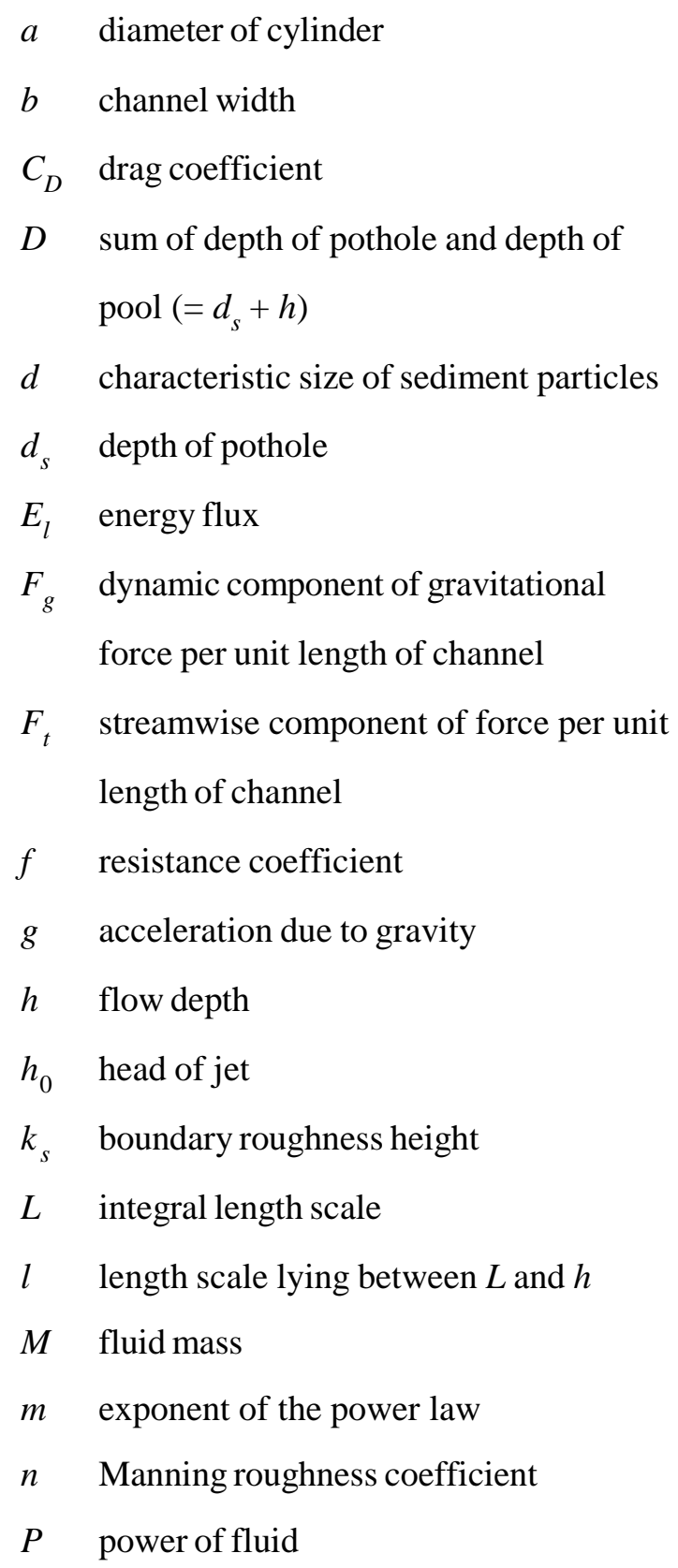

$q \quad$ discharge of jet per unit thickness

$R_{b} \quad$ hydraulic radius

Re Reynolds number $\left(=U_{m} R_{b} / v\right)$

$S_{0} \quad$ bottom slope of channel

$s_{l} \quad$ local stress

$\dot{s}_{l} \quad$ gradient of $s_{l}$

$t_{l} \quad$ circulation time

$t_{p} \quad$ TKE production rate

$U_{L} \quad$ velocity scale corresponding to $L$

$U_{m}$ mean velocity

$U_{\max }$ maximum velocity

$u_{*} \quad$ friction velocity

$\bar{u} \quad$ time-averaged flow velocity

$u^{+} \quad \bar{u} / u_{*}$

$\hat{u} \quad \bar{u} / U_{\max }$

$v_{l} \quad$ velocity scale corresponding to $l$

$v_{n} \quad$ velocity fluctuations normal to $W$

$v_{t} \quad$ velocity fluctuations tangential to $W$

$W \quad$ wetted surface

$z \quad$ vertical distance

$z_{0} \quad$ zero velocity level

$z^{+} \quad z / z_{0}$

$\hat{z} \quad z / \delta$

$\delta \quad$ boundary layer thickness

$\varepsilon \quad$ TKE dissipation rate

$\eta \quad$ Kolmogorov length scale

$\kappa \quad$ von Kármán constant

$\rho_{f} \quad$ mass density of fluid

$\rho_{s} \quad$ mass density of sediment particles 
$\tau_{c} \quad$ threshold boundary shear stress

$\tau_{t} \quad$ Reynolds shear stress

\section{References}

Bombardelli F A and Gioia G (2006) Scouring of granular beds by jet-driven axisymmetric turbulent cauldrons Physics of Fluids 18 088-101

Bradshaw P and Huang G P (1995) The law of the wall in turbulent flow Proceedings of the Royal Society of London A 451 (1941) 165-188

Carr M H (1979) Formation of Martian flood features by release of water from confined aquifers Journal of Geophysical Research 84 2995-3007

Dey S (2014) Fluvial hydrodynamics: Hydrodynamic and sediment transport phenomena Springer-Verlag

Frisch U (1995) Turbulence: The Legacy of A N Kolmogorov Cambridge University Press

George W K (2007) Is there a universal log law for turbulent wallbounded flows Philosophical Transactions of the Royal Society of London A $\mathbf{3 6 5}$ 789-806

Gioia G and Bombardelli F A (2002) Scaling and similarity in rough channel flows Physical Review Letters $\mathbf{8 8} 014501$

Gioia G and Bombardelli F A (2005) Localized turbulent flows on scouring granular beds Physical Review Letters 95 014501

Gioia G and Chakraborty P (2006) Turbulent friction in rough pipes and the energy spectrum of the phenomenological theory Physical Review Letters 96044502

Jiménez J (2004) Turbulent flows over rough walls Annual Review of Fluid Mechanics 36 173-196

Knight B and Sirovich L (1990) Kolmogorov inertial range for inhomogeneous turbulent flows Physical Review Letters 65 1356-1359

Kolmogorov A N (1941) The local structure of turbulence in incompressible viscous fluids at very large Reynolds numbers Doklady Akademii Nauk SSSR 30 299-303

Manes C and Brocchini M (2015) Local scour around structures and the phenomenology of turbulence Journal of Fluid Mechanics 779 309-324

Moser R D (1993) Kolmogorov inertial range spectra for inhomogeneous turbulence Physics of Fluids 6 794-801

Panton R L (2005) Review of wall turbulence as described by composite expansions Applied Mechanics Review 58 1-36

Pope S B (2000) Turbulent Flows Cambridge University Press

Raupach M R Antonia R A and Rajagopalan S (1991) Rough-wall turbulent boundary layers Applied Mechanics Review 44 $1-25$

Saddoughi S G and Veeravalli S V (1994) Local isotropy in turbulent boundary layers at high Reynolds number Journal of Fluid Mechanics 268333-372

Sreenivasan K R and Antonia R A (1997) The phenomenology of small-scale turbulence Annual Review of Fluid Mechanics 29 435-472

Schlichting H (1979) Boundary layer theory McGraw-Hill

Yen B C (1992) Dimensionally homogeneous Manning's formula Journal of Hydraulic Engineering 118 1326-1332. 